\title{
A Computational Model for Conversation Policies for Agent Communication
}

\author{
Jamal Bentahar ${ }^{1}$, Bernard Moulin ${ }^{1}$, John-Jules Ch. Meyer ${ }^{2}$, Brahim Chaib-draa ${ }^{1}$ \\ ${ }^{1}$ Laval University, Department of Computer Science and Software Engineering, Canada \\ jamal.bentahar.1@ulaval.ca \\ \{bernard.moulin, brahim.chaib-draa\}@ift.ulaval.ca \\ ${ }^{2}$ University Utrecht, Department of Computer Science, The Netherlands \\ $j j @ C s . u u . n 1$
}

\begin{abstract}
In this paper we propose a formal specification of a persuasion protocol between autonomous agents using an approach based on social commitments and arguments. In order to be flexible, this protocol is defined as a combination of a set of conversation policies. These policies are formalized as a set of dialogue games. The protocol is specified using two types of dialogue games: entry dialogue game and chaining dialogue games. The protocol terminates when exit conditions are satisfied. Using a tableau method, we prove that this protocol always terminates. The paper addresses also the implementation issues of our protocol using logical programming and an agentoriented platform.
\end{abstract}

\section{Introduction}

Research in agent communication has received much attention during the past years $[8,12,13]$. Agent communication protocols specify the rules of interaction governing a dialogue between autonomous agents in a multi-agent system. These protocols are patterns of behavior that restrict the range of allowed follow-up utterances at any stage during a dialogue. Unlike protocols used in distributed systems, agent communication protocols must take into account the fact that artificial agents are autonomous and proactive. These protocols must be flexible enough and must also be specified using a more expressive formalism than traditional formalisms such as finite state machines. Indeed, logic-based protocols seem an interesting way [15].

On the one hand, conversation policies [17] and dialogue games [11, 19] aim at offering more flexible protocols [18]. This is achieved by combining different policies and games to construct complete and more complex protocols. Conversation policies are declarative specifications that govern communication between autonomous agents. In this paper we propose to formalize these policies as a set of dialogue games. Dialogue games are interactions between players, in which each player moves by performing utterances according to a pre-defined set of roles. Indeed, protocols specified using finite state machines are not flexible in the sense that agents must respect the whole protocol from the beginning to the end. Thus, we propose to specify these protocols by small conversation policies that can be logically put together using 
a combination of dialogue games. On the other hand, in the last years, some research works addressed the importance of social commitments in agent communication [3, 4, $10,18,22,24]$. These works showed that social commitments are a powerful representation to model multi-agent interactions. Commitments provide a basis for a normative framework that makes it possible to model agents' communicative behaviors. This framework has the advantage of being expressive because all speech act types can be represented by commitments [10]. Commitment-based protocols enable the content of agent interactions to be represented and reasoned about $[16,25]$. In opposition to the BDI mental approach, the commitment-approach stresses the importance of conventions and the public aspects of dialogue. A speaker is committed to a statement when he makes this statement or when he agreed upon this statement made by another participant. In fact, we do not speak here about the expression of a belief, but rather about a particular relationship between a participant and a statement. What is important in this approach is not that an agent agrees or disagrees upon a statement, but rather the fact that the agent expresses agreement or disagreement, and acts accordingly.

In this paper we present a persuasion dialogue which is specified using conversation policies, dialogue games and a framework based on commitments. In addition, in order to allow agents to effectively reason on their communicative actions, our framework is also based on an argumentative approach. In our framework the agent's reasoning capabilities are linked to their ability to argue. In this paper we consider conversation policies as units specified by dialogue games whose moves are expressed in terms of actions that agents apply to commitments and arguments. Indeed, the paper presents three results: 1- A new formal language for specifying a persuasion dialogue as a combination of conversation policies. 2- A termination proof of the dialogue based on a tableau method [9]. 3- An implementation of the specification using an agent oriented and logical programming.

The paper is organized as follows. In Section 2, we introduce the main ideas of our approach based on commitments and arguments. In Section 3 we address the specification of our persuasion protocol based on this approach. We present the protocol form, the specification of each dialogue game and the protocol dynamics. We also present our termination proof. In Section 4 we describe the implementation of a prototype allowing us to illustrate how the specification of dialogue games is implemented. In Section 5 we compare our protocol to related work. Finally, in Section 6 we draw some conclusions and we identify some directions for future work.

\section{Commitment and Argument Approach}

\subsection{Social Commitments}

A social commitment $S C$ is a commitment made by an agent (the debtor), that some fact is true or that something will be done. This commitment is directed to a set of agents (creditors) [7]. A commitment is an obligation in the sense that the debtor must respect and behave in accordance with this commitment. In order to model the 
dynamics of conversations, we interpret a speech act $S A$ as an action performed on a commitment or on its content [3]. A speech act is an abstract act that an agent, the speaker, performs when producing an utterance $U$ and addressing it to another agent, the addressee. In the dialogue games that we specify in Section 3, the actions that an agent can perform on a commitment are: Act $\in\{$ Create, Withdraw $\}$. The actions that an agent can perform on a commitment content are: Act-content $\in\{$ Accept, Refuse, Challenge, Defend, Attack, Justify $\}$. In our framework, a speech act is interpreted either as an action applied to a commitment when the speaker is the debtor, or as an action applied to its content when the speaker is the debtor or the creditor [3]. Formally, a speech act can be defined as follows:

Definition 1. $S A\left(A g_{1}, A g_{2}, U\right)=_{\operatorname{def}} \operatorname{Act}\left(A g_{1}, S C\left(A g_{1}, A g_{2}, p\right)\right)$ | Act-content $\left(A g_{k}, S C\left(A g_{i}, A g_{j}, p\right)\right)$

where $i, j \in\{1,2\}$ and $(k=i$ or $k=j), p$ is the commitment content. The definiendum $S A\left(A g_{1}, A g_{2}, U\right)$ is defined by the definiens $\operatorname{Act}\left(A g_{1}, S C\left(A g_{1}, A g_{2}, p\right)\right)$ as an action performed by the debtor $A g_{1}$ on its commitment. The definiendum is defined by the definiens $A c t$-content $\left(A g_{k}, S C\left(A g_{i}, A g_{j}, p\right)\right)$ as an action performed by an agent $A g_{k}$ (the debtor or the creditor) on the commitment content.

\subsection{Argumentation and Social Commitments}

An argumentation system essentially includes a logical language $L$, a definition of the argument concept, and a definition of the attack relation between arguments. Several definitions were also proposed to define arguments. In our model, we adopt the following definitions from [14]. Here $\Gamma$ indicates a possibly inconsistent knowledge base with no deductive closure. F Stands for classical inference and $\equiv$ for logical equivalence.

Definition 2. An argument is a pair $(H, h)$ where $h$ is a formula of $L$ and $H$ a sub-set of $\Gamma$ such that : i) $H$ is consistent, ii) $H$ - $h$ and iii) $H$ is minimal, so no subset of $H$ satisfying both $i$ and ii exists. $H$ is called the support of the argument and $h$ its conclusion. We use the notation: $H=\operatorname{Support}(\mathrm{Ag}, h)$ to indicate that agent $\mathrm{Ag}$ has a support $H$ for $h$.

Definition 3. Let $\left(H_{1}, h_{1}\right),\left(H_{2}, h_{2}\right)$ be two arguments. $\left(H_{1}, h_{1}\right)$ attacks $\left(H_{2}, h_{2}\right)$ iff $h_{1} \equiv$ $\neg h_{2}$.

In fact, before committing to some fact $h$ being true (i.e. before creating a commitment whose content is $h$ ), the speaker agent must use its argumentation system to build an argument $(H, h)$. On the other side, the addressee agent must use its own argumentation system to select the answer it will give (i.e. to decide about the appropriate manipulation of the content of an existing commitment). For example, an agent $A g_{l}$ accepts the commitment content $h$ proposed by another agent if $A g_{l}$ has an argument for $h$. If $A g_{l}$ has an argument neither for $h$, nor for $\neg h$, then it challenges $h$.

In our framework, we distinguish between arguments that an agent has (private arguments) and arguments that this agent used in its conversation (public arguments). 
Thus, we use the notation: $S=$ Create_Support $\left(A g, S C\left(A g_{1}, A g_{2}, p\right)\right)$ to indicate the set of commitments $S$ created by agent $A g$ to support the content of $S C\left(A g_{1}, A g_{2}, p\right)$. This support relation is transitive i.e.:

$\left(S C\left(A g_{1}, A g_{2}, p_{2}\right) \in\right.$ Create_Support $\left(A g, S C\left(A g_{1}, A g_{2}, p_{1}\right)\right)$

$\wedge S C\left(A g_{1}, A g_{2}, p_{1}\right) \in$ Create_Support $\left.\left(A g, S C\left(A g_{1}, A g_{2}, p_{0}\right)\right)\right)$

$\Rightarrow S C\left(A g_{1}, A g_{2}, p_{2}\right) \in$ Create_Support $\left(A g, S C\left(A g_{1}, A g_{2}, p_{0}\right)\right)$

Other details about our commitment and argument approach are described in [3]. Surely, an argumentation system is essential to help agents to act on commitments and on their contents. However, reasoning on other social attitudes should be taken into account in order to explain the agents' decisions. In our persuasion protocol we use the agents' trustworthiness to decide, in some cases, about the acceptance of arguments [5].

\section{Conversation Policies for Persuasion Dialogue}

\subsection{Protocol Form}

Our persuasion protocol is specified as a set of conversation policies. In order to be flexible, these policies are defined as initiative/reactive dialogue games. In accordance with our approach, the game moves are considered as actions that agents apply to commitments and to their contents. A dialogue game is specified as follows:

$$
\text { Action_Ag } \stackrel{\text { Cond }}{\longrightarrow} \text { Action_Ag }{ }_{2}
$$

This specification indicates that if an agent $A g_{1}$ performs the action $A c t i o n \_A g_{1}$, and that the condition Cond is satisfied, then the interlocutor $\mathrm{Ag}_{2}$ will perform the action Action_Ag $\mathrm{Ag}_{2}$. The condition Cond is expressed in terms of the possibility of generating an argument from the agent's argumentation system and in terms of the interlocutor's trustworthiness. Because we suppose that we have always two agents $A g_{I}$ and $A g_{2}$, a SC whose content is $p$ will be denoted in the rest of this paper $S C(p)$. We use the notation: $p \triangle A r g \_S y s\left(A g_{l}\right)$ to denote the fact that a propositional formula $p$ can be generated from the argumentation system of $A g_{l}$ denoted $\operatorname{Arg} \operatorname{Sys}_{-}\left(A g_{l}\right)$. The formula $\neg\left(p \triangle A r g \_S y s\left(A g_{l}\right)\right)$ indicates the fact that $p$ cannot be generated from $A g_{I}$ 's argumentation system. A propositional formula $p$ can be generated from an agent's argumentation system, if this agent can find an argument that supports $p$. To simplify the formalism, we use the notation $A c t^{\prime}(A g, S C(p))$ to indicate the action that agent $A g$ performs on the commitment $S C(p)$ or on its content (Act' $\in\{$ Create, Withdraw, Accept, Challenge, Refuse $\}$ ). For the actions related to the argumentation relations, we write $\operatorname{Act}-\operatorname{Arg}(A g,[S C(q)], S C(p))$. This notation indicates that $A g$ defends (resp. attacks or justifies) the content of $S C(p)$ by the content of $S C(q)$ (Act-Arg $\in\{$ Defend, Attack, Justify\}). In a general way, we use the notation $A_{c t}^{\prime}(A g, S)$ to indicate the action that $\mathrm{Ag}$ performs on the set of commitments $S$ or on the contents of these commitments, and the notation $\operatorname{Act}-\operatorname{Arg}(A g$, [S], $S C(p))$ to indicate the argumentationrelated action that $A g$ performs on the content of $S C(p)$ using the contents of $S$ as 
support. We also introduce the notation $\operatorname{Act}-\operatorname{Arg}\left(\operatorname{Ag},[S], S^{\prime}\right)$ to indicate that $A g$ performs an argumentation-related action on the contents of a set of commitments $S^{\text {' }}$ using the contents of $S$ as supports.

We distinguish two types of dialogue games: entry game and chaining games. The entry game allows the two agents to open the persuasion dialogue. The chaining games make it possible to construct the conversation. The protocol terminates when the exit conditions are satisfied (Fig. 1).

$\longrightarrow$ Entry game $\longrightarrow$ Chaining games

Fig. 1. The general form of the protocol

\subsection{Dialogue Games Specification}

\section{A Entry Game}

The conversational policy that describes the entry conditions in our persuasion protocol about a propositional formula $p$ is described by the entry dialogue game as follows (Specification 1):

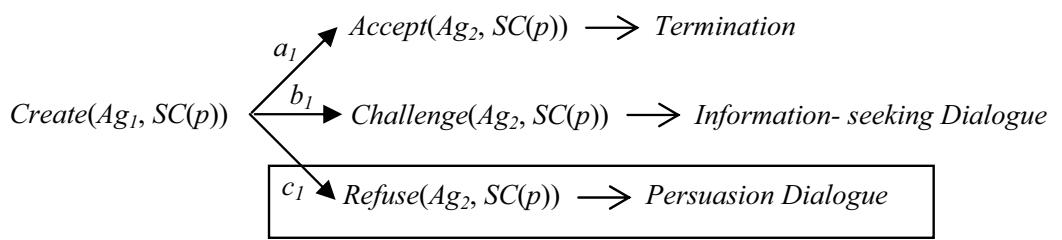

where $a_{1}, b_{1}$ and $c_{1}$ are three conditions specified as follows:

$a_{1}=p \triangle A r g \_$Sys $\left(\mathrm{Ag}_{2}\right)$

$b_{1}=\neg\left(p \triangle A r g \_\operatorname{Sys}\left(A g_{2}\right)\right) \wedge \neg\left(\neg p \triangle A r g \_\operatorname{Sys}\left(A g_{1}\right)\right)$

$c_{1}=\neg p \triangle \operatorname{Arg} \_$Sys $\left(\mathrm{Ag}_{2}\right)$

If $\mathrm{Ag}_{2}$ has an argument for $p$ then it accepts $p$ (the content of $S C(p)$ ) and the conversation terminates as soon as it begins (Condition $a_{1}$ ). If $A g_{2}$ has neither an argument for $p$ nor for $\neg p$, then it challenges $p$ and the two agents open an information-seeking dialogue (condition $b_{1}$ ). The persuasion dialogue starts when $\mathrm{Ag}_{2}$ refuses $p$ because it has an argument against $p$ (condition $c_{l}$ ).

\section{B Defense Game}

Once the two agents opened a persuasion dialogue, the initiator must defend its point of view. Thus, it must play a defense game. Our protocol is specified in such a way that the persuasion dynamics starts by playing a defense game. We have (Specification 2): 


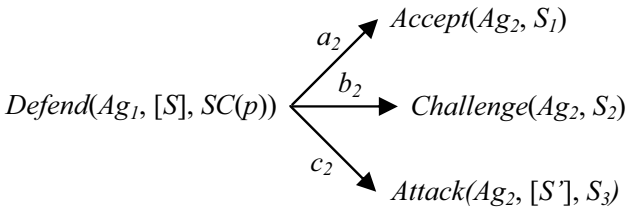

where:

$S=\left\{S C\left(p_{i}\right) / i=0, \ldots, n\right\}, p_{i}$ are propositional formulas.

$\mathrm{Y}_{i=1}^{3} S_{i}=S, S_{i \mathrm{I}} S_{j}=\varnothing, i, j=1, \ldots, 3 \& i \neq j$

By definition, Defend $\left(A g_{l}\right.$, $\left.[S], S C(p)\right)$ means that $A g_{l}$ creates $S$ in order to defend the content of $S C(p)$. Formally:

$\operatorname{Defend}\left(A g_{1},[S], S C(p)\right)=_{\text {def }}\left(C r e a t e\left(A g_{1}, S\right) \wedge S=\right.$ Create_Support $\left.\left(A g_{1}, S C(p)\right)\right)$

We consider this definition as an assertional description of the Defend action. We propose similar definitions for Attack and Justify actions which are not presented in this paper.

This specification indicates that according to the three conditions $\left(a_{2}, b_{2}\right.$ and $\left.c_{2}\right)$, $\mathrm{Ag}_{2}$ can accept a subset $S_{1}$ of $S$, challenge a subset $S_{2}$ and attack a third subset $S_{3}$. Sets $S_{i}$ and $S_{j}$ are mutually disjoint because $A g_{2}$ cannot, for example, both accept and challenge the same commitment content. Accept, Challenge and Attack a set of commitment contents are defined as follows:

$\operatorname{Accept}\left(A g_{2}, S_{1}\right)=_{\text {def }}\left(\forall i, S C\left(p_{i}\right) \in S_{1} \Rightarrow \operatorname{Accept}\left(A g_{2}, S C\left(p_{i}\right)\right)\right)$

Challenge $\left(A g_{2}, S_{2}\right)=_{\text {def }}\left(\forall i, S C\left(p_{i}\right) \in S_{2} \Rightarrow\right.$ Challenge $\left.\left(A g_{2}, S C\left(p_{i}\right)\right)\right)$

$\operatorname{Attack}\left(A g_{2},\left[S^{\prime}\right], S_{3}\right)=_{\text {def }} \forall i, S C\left(p_{i}\right) \in S_{3} \Rightarrow \exists S_{j}^{\prime} \subseteq S^{\prime}, \operatorname{Attack}\left(A g_{2},\left[S_{j}^{\prime}\right], S C\left(p_{i}\right)\right)$

where: $\mathrm{Y}_{j=0}^{m} S_{j}^{\prime}=S^{\prime}$. This indication means that any element of $S^{\prime}$ is used to attack one or more elements of $S_{3}$.

The conditions $a_{2}, b_{2}$ and $c_{2}$ are specified as follows:

$a_{2}=\forall i, S C\left(p_{i}\right) \in S_{1} \Rightarrow p_{i} \triangle \operatorname{Arg} \operatorname{Sys}_{-}\left(\mathrm{Ag}_{2}\right)$

$b_{2}=\forall i, S C\left(p_{i}\right) \in S_{2} \Rightarrow\left(\neg\left(p_{i} \triangle \operatorname{Arg} \_\operatorname{Sys}\left(A g_{2}\right)\right) \wedge \neg\left(\neg p_{i} \triangle \operatorname{Arg} \operatorname{Sys}\left(A g_{2}\right)\right)\right)$

$c_{2}=\forall i, S C\left(p_{i}\right) \in S_{3} \Rightarrow \exists S_{j}^{\prime} \subseteq S^{\prime}, \operatorname{Content}\left(S_{j}^{\prime}\right)=\operatorname{Support}\left(A g_{2}, \neg p_{i}\right)$

where Content $\left(S_{j}^{\prime}\right)$ indicates the set of contents of the commitments $S_{j}$.

\section{Challenge Game}

The challenge game is specified as follows (Specification 3):

$$
\text { Challenge }\left(A g_{1}, S C(p)\right) \stackrel{a_{3}}{\longrightarrow} \operatorname{Justify}\left(A g_{2},[S], S C(p)\right)
$$

where the condition $a_{3}$ is specified as follows:

$a_{3}=\left(\operatorname{Content}(S)=\operatorname{Support}\left(A g_{2}, p\right)\right)$

In this game, the condition $a_{3}$ is always true. The reason is that in accordance with the commitment semantics, an agent must always be able to defend the commitment it created [4]. 


\section{Justification Game}

For this game we distinguish two cases:

Case1. $S C(p) \notin S$

In this case, $A g_{1}$ justifies the content of its commitment $S C(p)$ by creating a set of commitments $S$. As for the Defend action, $A g_{2}$ can accept, challenge and/or attack a subset of $S$. The specification of this game is as follows (Specification 4):

where:

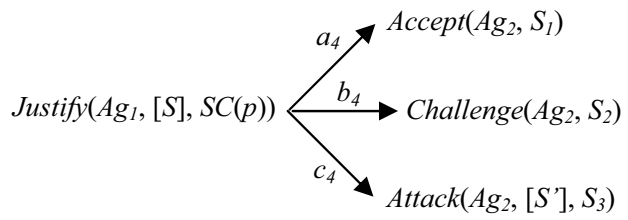

$S=\left\{S C\left(p_{i}\right) / i=0, \ldots, n\right\}, p_{i}$ are propositional formulas.

$\mathrm{Y}_{i=1}^{3} S_{i}=S, S_{i \mathrm{I}} S_{j}=\varnothing, i, j=1, \ldots, 3 \& i \neq j$

$a_{4}=a_{2}, b_{4}=b_{2}, c_{4}=c_{2}$

Case2. $\{S C(p)\}=S$

In this case, the justification game has the following specification (Specification 5):

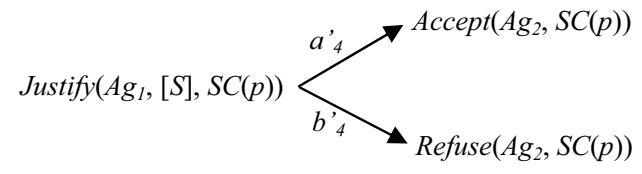

$A g_{I}$ justifies the content of its commitment $S C(p)$ by itself (i.e. by $p$ ). This means that $p$ is part of $A g_{1}$ 's knowledge. Only two moves are possible for $\left.A g_{2}: 1\right)$ accept the content of $S C(p)$ if $A g_{1}$ is a trustworthy agent for $\left.A g_{2}\left(a^{\prime}{ }_{4}\right), 2\right)$ if not, refuse this content $\left(b_{4}^{\prime}\right) . A g_{2}$ cannot attack this content because it does not have an argument against $p$. The reason is that $A g_{1}$ plays a justification game because $A g_{2}$ played a challenge game.

\section{E Attack Game}

The attack game is specified as follows (Specification 6):

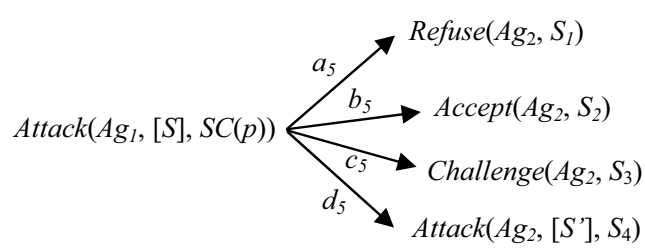

where:

$S=\left\{S C\left(p_{i}\right) / i=0, \ldots, n\right\}, p_{i}$ are propositional formulas.
$\mathrm{Y}_{i=1}^{4} S_{i}=S, \operatorname{Card}\left(S_{I}\right)=1, S_{i \mathrm{I}} S_{j}=\varnothing, i, j=1, \ldots, 4 \& i \neq j$ 
The conditions $a_{5}, b_{5}, c_{5}$ and $d_{5}$ are specified as follows:

$a_{5}=\exists i, S C\left(p_{i}\right) \in$ Create_Support $\left(A g_{2}, S C(\neg q)\right)$

where $S_{1}=\{S C(q)\}$

$b_{5}=\forall i, S C\left(p_{i}\right) \in S_{2} \Rightarrow p_{i} \triangle A r g \_S y s\left(A_{2}\right)$

$c_{5}=\forall i, S C\left(p_{i}\right) \in S_{3} \Rightarrow\left(\neg\left(p_{i} \bullet A r g \_S y s\left(A g_{2}\right)\right) \wedge \neg\left(\neg p_{i} \triangle A r g \_S y s\left(A g_{2}\right)\right)\right)$

$d_{5}=\forall i, S C\left(p_{i}\right) \in S_{4} \Rightarrow \exists S_{j}^{\prime} \subseteq S^{\prime}$,

$\operatorname{Content}\left(S_{j}^{\prime}\right)=\operatorname{Support}\left(A g_{2}, \neg p_{i}\right) \wedge \nexists k, S C\left(p_{k}\right) \in$ Create_Support $\left(A g_{2}, S C\left(\neg p_{i}\right)\right)$

$A g_{2}$ refuses $A g_{1}$ 's argument if $A g_{2}$ already attacked this argument. In other words, $\mathrm{Ag}_{2}$ refuses $\mathrm{Ag}_{1}$ 's argument if $\mathrm{Ag}_{2}$ cannot attack this argument since it already attacked it, and it cannot accept it or challenge it since it has an argument against this argument. We have only one element in $S_{I}$ because we consider a refusal move as an exit condition. The acceptance and the challenge actions of this game are the same as the acceptance and the challenge actions of the defense game. Finally, $A g_{2}$ attacks $\mathrm{Ag}_{1}$ 's argument if $\mathrm{Ag}_{2}$ has an argument against $A g_{1}$ 's argument, and if $\mathrm{Ag}_{2}$ did not attack $A g_{1}$ 's argument before. In $d_{5}$, the universal quantifier means that $A g_{2}$ attacks all $A g_{1}$ 's arguments for which it has an against-argument. The reason is that $A g_{2}$ must act on all commitments created by $A g_{1}$. The temporal aspect (the past) of $a_{5}$ and $d_{5}$ is implicitly integrated in Create_Support $\left(\mathrm{Ag}_{2}, S C(\neg q)\right)$ and Create_Support $\left(\mathrm{Ag}_{2}\right.$, $\left.S C\left(\neg p_{i}\right)\right)$.

\section{F Termination}

The protocol terminates either by a final acceptance or by a refusal. There is a final acceptance when $A g_{2}$ accepts the content of the initial commitment $S C(p)$ or when $A g_{1}$ accepts the content of $S C(\neg p) . A g_{2}$ accepts the content of $S C(p)$ iff it accepts all the supports of $S C(p)$. Formally:

$\operatorname{Accept}\left(\mathrm{Ag}_{2}, S C(p)\right) \Leftrightarrow$

$\left[\forall i, S C\left(p_{i}\right) \in\right.$ Create_Support $\left.\left(A g_{1}, S C(p)\right) \Rightarrow \operatorname{Accept}\left(A g_{2}, S C\left(p_{i}\right)\right)\right]$

The acceptance of the supports of $S C(p)$ by $\mathrm{Ag}_{2}$ does not mean that they are accepted directly after their creation by $A g_{l}$, but it can be accepted after a number of challenge, justification and attack games. When $\mathrm{Ag}_{2}$ accepts definitively, then it withdraws all commitments whose content was attacked by $\mathrm{Ag}_{1}$. Formally:

$\operatorname{Accept}\left(A g_{2}, S C(p)\right) \Rightarrow\left[\forall i, \forall S, \operatorname{Attack}\left(A g_{1},[S], S C\left(p_{i}\right)\right) \Rightarrow \operatorname{Withdraw}\left(\operatorname{Ag}_{2}, S C\left(p_{i}\right)\right)\right]$

On the other hand, $\mathrm{Ag}_{2}$ refuses the content of $S C(p)$ iff it refuses one of the supports of $S C(p)$. Formally:

$\operatorname{Refuse}\left(A g_{2}, S C(p)\right) \Leftrightarrow\left[\exists i, S C\left(p_{i}\right) \in\right.$ Create_Support $\left.\left(A g_{1}, S C(p)\right) \wedge \operatorname{Refuse}\left(A g_{2}, S C\left(p_{i}\right)\right)\right]$

\subsection{Protocol Dynamics}

The persuasion dynamics is described by the chaining of a finite set of dialogue games: acceptance move, refusal move, defense, challenge, attack and justification games. These games can be combined in a sequential and parallel way (Fig. 2).

After $A g_{1}$ 's defense game at moment $t_{1}, A g_{2}$ can, at moment $t_{2}$, accept a part of the arguments presented by $A g_{l}$, challenge another part, and/or attack a third part. These 
games are played in parallel. At moment $t_{3}, A g_{1}$ answers the challenge game by playing a justification game and answers the attack game by playing an acceptance move, a challenge game, another attack game, and/or a final refusal move. The persuasion dynamics continues until the exit conditions become satisfied (final acceptance or a refusal). From our specifications, it follows that our protocol plays the role of the dialectical proof theory of the argumentation system.

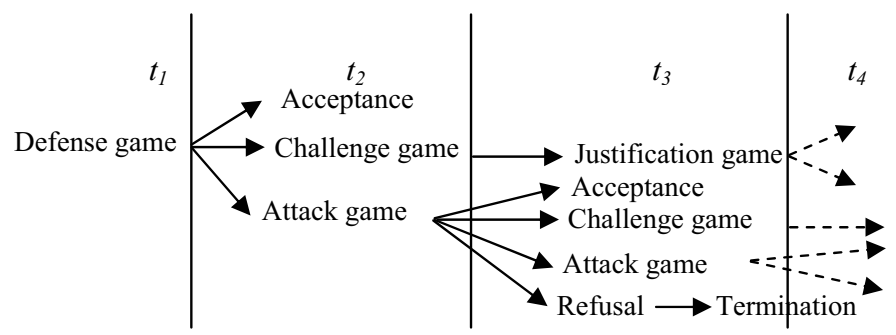

Fig. 2. The persuasion dialogue dynamics

Indeed, our persuasion protocol can be described by the following BNF grammar: Persuasion protocol: Defense game $\sim$ Dialogue games

Dialogue games: (Acceptance move

$$
\begin{aligned}
& \text { // (Challenge game } \sim \text { Justification game } \sim \text { Dialogue games }) \\
& \text { // (Attack game } \sim \text { Dialogue games)) } \\
& \text { | refusal move }
\end{aligned}
$$

where: " $\sim$ " is the sequencing symbol, "/// is the possible parallelization symbol. Two games Gamel and Game 2 are possibly parallel (i.e. Game1 // Game2) iff an agent can play the two games in parallel or only one game (Game1 or Game2).

\subsection{Termination proof}

Theorem. The protocol dynamics always terminates.

Proof. To prove this theorem, we use a tableau method [9]. The idea is to formalize our specifications as tableau rules and then to prove the finiteness of the tableau. Tableau rules are written in such a way that premises appear above conclusions. Using a tableau method means that the specifications are conducted in a top-down fashion. For example, specification 2 (p 3.2 ) can be expressed by the following rules:

$$
\begin{gathered}
R 1: \frac{\operatorname{Defend}\left(A g_{1},[S], S C(p)\right)}{\operatorname{Accept}\left(A g_{2}, S_{1}\right)} R 2: \frac{\operatorname{Defend}\left(A g_{1},[S], S C(p)\right)}{\operatorname{Challenge}\left(A g_{2}, S_{1}\right)} \\
R 3: \frac{\operatorname{Defend}\left(A g_{1},[S], S C(p)\right)}{\operatorname{Attack}\left(A g_{2},\left[S^{\prime}\right], S_{1}\right)}
\end{gathered}
$$

We denote the formulas of our specifications by $\sigma$, and we define $E$ the set of $\sigma$. We define an ordering $\pi$ on $E$ that has no infinite ascending chains. Intuitively, this 
relation is to hold between $\sigma_{1}$ and $\sigma_{2}$ if it is possible that $\sigma_{1}$ is an ancestor of $\sigma_{2}$ in some tableau. Before defining this ordering, we introduce some notations: $A c t *(A g$, $\left.[S], S^{\prime}\right)$ with $A c t^{*} \in\left\{A c t^{\prime}, A c t-A r g\right\}$ is a formula. We notice that formulas in which there is no support [S], can be written as follows: $A c t^{*}\left(A g,[\phi], S^{\prime}\right) . \sigma[S] \rightarrow_{R} \sigma\left[S^{\prime}\right]$ indicates that the tableau rule $R$ has the formula $\sigma[S]$ as premise and the formula $\sigma\left[S^{\prime}\right]$ as conclusion, with $\sigma[S]=A c t^{*}\left(A g,[S], S^{\prime}\right)$. The size $|S|$ is the number of commitments in $S$.

Definition4. Let $\sigma\left[S_{i}\right]$ be a formula and $E$ the set of $\sigma\left[S_{i}\right]$. The ordering $\pi$ on $E$ is defined as follows. We have $\sigma\left[S_{0}\right] \pi \sigma\left[S_{l}\right]$ if:

$\left|S_{I}\right|<\left|S_{0}\right|$ or

For all rules Ri such that $\sigma\left[S_{I}\right] \rightarrow_{R I} \sigma\left[S_{2}\right] \ldots \rightarrow_{R n} \sigma\left[S_{n}\right]$ we have $\left|S_{n}\right|=0$.

Intuitively, in order to prove that a tableau system is finite, we need to prove the following:

1- if $\sigma\left[S_{0}\right] \rightarrow_{R} \sigma\left[S_{1}\right]$ then $\sigma\left[S_{0}\right] \pi \sigma\left[S_{1}\right]$.

2- $\pi$ has no infinite ascending chains (i.e. the inverse of $\pi$ is well-founded).

Property 1 reflects the fact that applying tableau rules results in shorter formulas, and property 2 means that this process has a limit. The proof of 1 proceeds by a case analysis on $R$. Most cases are straightforward; we consider here the case of $R 3$. For this rule we have two cases. If $\left|S_{l}\right|<\left|S_{0}\right|$, then $\sigma\left[S_{0}\right] \pi \sigma\left[S_{l}\right]$. If $\left|S_{l}\right| \geq\left|S_{0}\right|$, the rules corresponding to the attack specification can be applied. The three first rules are straightforward since $S_{2}=\phi$. For the last rule, we have the same situation that $R 3$. Suppose that there is no path in the tableau $\sigma\left[S_{1}\right] \rightarrow_{R 1} \sigma\left[S_{2}\right] \ldots \rightarrow_{R n} \sigma\left[S_{n}\right]$ such that $\left|S_{n}\right|=0$. This means that the number of arguments that agents have is infinite or that one or several arguments are used several times. However, these two situations are not possible.

From the definition of $\pi$ and since $\left|S_{0}\right| \in N(<\infty)$ and $<$ is well-founded in $N$, it follows that there is no infinite ascending chains of the form $\sigma\left[S_{0}\right] \pi \sigma\left[S_{I}\right] \ldots$

\section{Implementation}

In this section we describe the implementation of the different dialogue games using the $J_{a c k}{ }^{T M}$ platform [23]. We chose this language for three main reasons:

1 - It is an agent-oriented language offering a framework for multi-agent system development. This framework can support different agent models.

2- It is built on top of and fully integrated with the Java programming language. It includes all components of Java and it offers specific extensions to implement agents' behaviors.

3- It supports logical variables and cursors. These features are particularly helpful when querying the state of an agent's beliefs. Their semantics is mid-way between logic programming languages with the addition of type checking Java style and embedded SQL. 


\subsection{General Architecture}

Our system consists of two types of agents: conversational agents and trust model agents. These agents are implemented as Jack ${ }^{T M}$ agents, i.e. they inherit from the basic class Jack ${ }^{T M}$ Agent. Conversational agents are agents that take part in the persuasion dialogue. Trust model agents are agents that can inform an agent about the trustworthiness of another agent.

According to the specification of the justification game, an agent $\mathrm{Ag}_{2}$ can play an acceptance or a refusal move according to whether it considers that its interlocutor $\mathrm{Ag}_{1}$ is trustworthy or not. If $\mathrm{Ag}_{1}$ is unknown for $\mathrm{Ag}_{2}, \mathrm{Ag}_{2}$ can ask agents that it considers trustworthy for it to offer a trustworthiness assessment of $A g_{1}$. From the received answers, $\mathrm{Ag}_{2}$ can build a trustworthiness graph and measure the trustworthiness of $A g_{1}$. This trustworthiness model is described in detail in [5].

\subsection{Implementation of the Dialogue Games}

To be able to take part in a persuasion dialogue, agents must possess knowledge bases that contain arguments. In our system, these knowledge bases are implemented as Jack ${ }^{T M}$ beliefsets. Beliefsets are used to maintain an agent's beliefs about the world. These beliefs are represented in a first order logic and tuple-based relational model. The logical consistency of the beliefs contained in a beliefset is automatically maintained. The advantage of using beliefsets over normal Java data structures is that beliefsets have been specifically designed to work within the agent-oriented paradigm.

Our knowledge bases (KBs) contain two types of information: arguments and beliefs. Arguments have the form ([Support], Conclusion), where Support is a set of propositional formulas and Conclusion is a propositional formula. Beliefs have the form ([Belief], Belief) i.e. Support and Conclusion are identical. The meaning of the propositional formulas (i.e. the ontology) is recorded in a beliefset whose access is shared between the two agents.

To open a dialogue game, an agent uses its argumentation system. The argumentation system allows this agent to seek in its knowledge base an argument for a given conclusion or for its negation ("against argument"). For example, before creating a commitment $S C(p)$, an agent must find an argument for $p$. This enables us to respect the commitment semantics by making sure that agents can always defend the content of their commitments.

Agent communication is done by sending and receiving messages. These messages are events that extend the basic Jack ${ }^{T M}$ event: MessageEvent class. MessageEvents represent events that are used to communicate with other agents. Whenever an agent needs to send a message to another agent, this information is packaged and sent as a MessageEvent. A MessageEvent can be sent using the primitive: Send(Destination, Message). In our protocol, Message represents the action that an agent applies to a commitment or to its content, for example: $\operatorname{Create}\left(A g_{l}, S C(p)\right)$, etc.

Our dialogue games are implemented as a set of events (MessageEvents) and plans. A plan describes a sequence of actions that an agent can perform when an event occurs. Whenever an event is posted and an agent chooses a task to handle it, the first 
thing the agent does is to try to find a plan to handle the event. Plans are methods describing what an agent should do when a given event occurs.

Each dialogue game corresponds to an event and a plan. These games are not implemented within the agents' program, but as event classes and plan classes that are external to agents. Thus, each conversational agent can instantiate these classes. An agent $A g_{1}$ starts a dialogue game by generating an event and by sending it to its interlocutor $\mathrm{Ag}_{2} . \mathrm{Ag}_{2}$ executes the plan corresponding to the received event and answers by generating another event and by sending it to $A g_{1}$. Consequently, the two agents can communicate by using the same protocol since they can instantiate the same classes representing the events and the plans. For example, the event Event_Attack_Commitment and the plan Plan_ev_Attack_commitment implement the defense game. The architecture of our conversational agents is illustrated in Fig. 3.

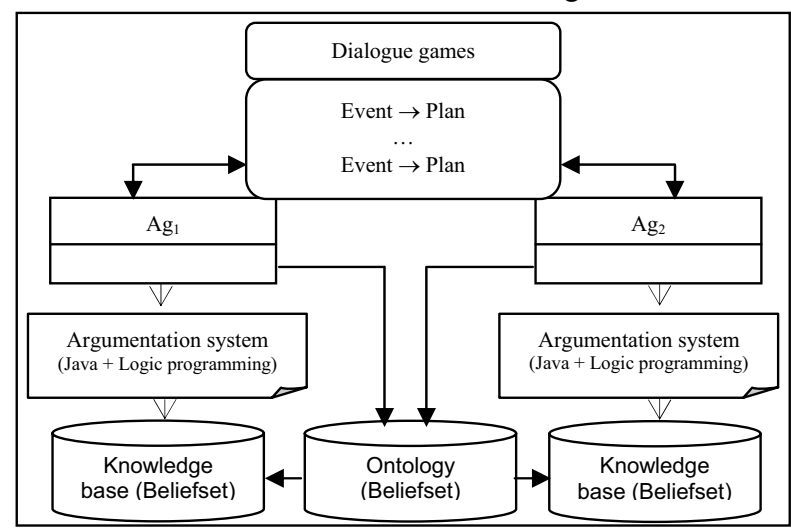

Fig. 3. The architecture of conversational agents

To start the entry game, an agent (initiator) chooses a goal that it tries to achieve. This goal is to persuade its interlocutor that a given propositional formula is true. For this reason, we use a particular event: BDI Event (Belief-Desire-Intention). BDI events model goal-directed behavior in agents, rather than plan-directed behavior. What is important is the desired outcome, not the method chosen to achieve it. This type of events allows an agent to pursue long term goals.

\subsection{Example}

In this section we present a simple example dialogue that illustrates some notions presented in this paper. This example was also studied in [2] in a context of strategical considerations for argumentative agents. The letters on the left of the utterances are the propositional formulas that represent the propositional contents. The $A g_{I}$ 's KB

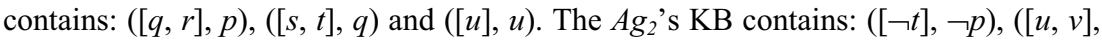
$\neg t),([u], u)$ and $([v], v)$. The combination of the dialogue games that allows us to describe the persuasion dialogue dynamics is as follows: 
$\mathrm{Ag}_{1}$ : Newspapers can publish information I $(p)$.

$\mathrm{Ag}_{2}$ : I don't agree with you.

$\mathrm{Ag}_{1}$ : They can publish information I because it is not private $(q)$, and any public information can be published $(r)$.

$\mathrm{Ag}_{2}$ : Why is information I public?

$\mathrm{Ag}_{1}$ : Because it concerns a Minister $(s)$, and information concerning a Minister are public $(t)$.

$\mathrm{Ag}_{2}$ : Information concerning a Minister is not necessarily public, because information I is about the health of Minister $(u)$, and information about the health remains private $(v)$.

$\mathrm{Ag}_{1}$ : I accept your argument.

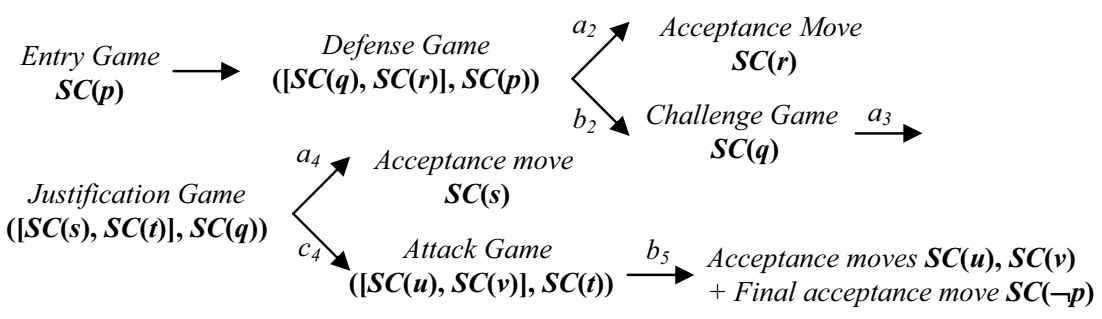

$A g_{1}$ creates $S C(p)$ to achieve the goal of persuading $A g_{2}$ that $p$ is true. $A g_{1}$ can create this commitment because it has an argument for $p . A g_{2}$ refuses $S C(p)$ because it has an argument against $p$. Thus, the entry game is played and the persuasion dialogue is opened. $A g_{1}$ defends $S C(p)$ by creating $S C(q)$ and $S C(r)$. $A g_{2}$ accepts $S C(r)$ because it has an argument for $r$ and challenges $S C(q)$ because it has argument neither for $q$ nor against $q . A g_{1}$ plays a justification game to justify $S C(q)$ by creating $S C(s)$ and $S C(t) . A g_{2}$ accepts the content of $S C(s)$ and attack the content of $S C(t)$ by creating $S C(u)$ and $S C(v)$. Finally, $A g_{1}$ plays acceptance moves because it has an argument for $u$ and it does not have arguments against $v$ and the dialogue terminates. Indeed, before accepting $v, A g_{1}$ challenges it and $A g_{2}$ defends it by itself (i.e. ([SC(v), $\left.\left.S C(v)\right]\right)$ ). Ag1 updates its $\mathrm{KB}$ by including the new argument.

\section{Related Work}

In this section, we compare our protocol with some proposals that have been put forward in two domains: dialogue modeling and commitment based protocols.

1- Dialogue modeling. In [1] and [20] Amgoud, Parsons and their colleagues studied argumentation-based dialogues. They proposed a set of atomic protocols which can be combined. These protocols are described as a set of dialogue moves using Walton and Krabbe's classification and formal dialectics. In these protocols, agents can argue about the truth of propositions. Agents can communicate both propositional statements and arguments about these statements. These protocols have the advantage of taking into account the capacity of agents to reason as well as their attitudes (confident, careful, etc.). In addition, Prakken [21] proposed a framework for 
protocols for dynamic disputes, i.e., disputes in which the available information can change during the conversation. This framework is based on a logic of defeasible argumentation and is formulated for dialectical proof theories. Soundness and completeness of these protocols have also been studied. In the same direction, Brewka [6] developed a formal model for argumentation processes that combines nonmonotonic logic with protocols for dispute. Brewka pays more attention to the speech act aspects of disputes and he formalizes dispositional protocols in situation calculus. Such a logical formalization of protocols allows him to define protocols in which the legality of a move can be disputed. Semantically, Amgoud, Parsons, Prakken and Brewkas' approaches use a defeasible logic. Therefore, it is difficult, if not impossible, to formally verify the proposed protocols.

There are many differences between our protocol and the protocols proposed in the domain of dialogue modeling: 1. Our protocol uses not only an argumentative approach, but also a public one. Locutions are formalized not as agents' private attitudes (beliefs, intentions, etc.), but as social commitments. In opposition of private mental attitudes, social commitments can be verified. 2. Our protocol is based on a combination of dialogue games instead of simple dialogue moves. Using our dialogue game specifications enables us to specify the entry and the exit conditions more clearly. In addition, computationally speaking, dialogue games provide a good balance between large protocols that are very rigid and atomic protocols that are very detailed. 3. From a theoretical point of view, Amgoud, Parsons, Prakken and Brewkas' protocols use moves from formal dialectics, whereas our protocol uses actions that agents apply on commitments. These actions capture the speech acts that agents perform when conversing (see Definition 1). The advantage of using these actions is that they enable us to better represent the persuasion dynamics considering that their semantics is defined in an unambiguous way in a temporal and dynamic logic [4]. Specifying protocols in this logic allows us to formally verify these protocols using model checking techniques. 4. Amgoud, Parsons and Prakkens' protocols use only three moves: assertion, acceptance and challenge, whereas our protocol uses not only creation, acceptance, refusal and challenge actions, but also attack and defense actions in an explicit way. These argumentation relations allow us to directly illustrate the concept of dispute in this type of protocols. 5. Amgoud, Parsons, Prakken and Brewka use an acceptance criterion directly related to the argumentation system, whereas we use an acceptance criteria for conversational agents (supports of arguments and trustworthiness). This makes it possible to decrease the computational complexity of the protocol for agent communication.

2- Commitment-based protocols. Yolum and Singh [25] developed an approach for specifying protocols in which actions' content is captured through agents' commitments. They provide operations and reasoning rules to capture the evolution of commitments. In a similar way, Fornara and Colombetti [16] proposed a method to define interaction protocols. This method is based on the specification of an interaction diagram (ID) specifying which actions can be performed under given conditions. These approaches allow them to represent the interaction dynamics through the allowed operations. Our protocol is comparable to these protocols because it is also based on commitments. However, it is different in the following respects. The choice of the various operations is explicitly dealt with in our protocol by using argumentation and trustworthiness. In commitment-based protocols, there is 
no indication about the combination of different protocols. However, this notion is essential in our protocol using dialogue games. Unlike commitment-based protocols, our protocol plays the role of the dialectical proof theory of an argumentation system. This enables us to represent different dialogue types as studied in the philosophy of language. Finally, we provide a termination proof of our protocol whereas this property is not yet studied in classical commitment-based protocols.

\section{Conclusion and Future Work}

The contribution of this paper is the proposition of a logical language for specifying persuasion protocols between agents using an approach based on commitments and arguments. This language has the advantage of expressing the public elements and the reasoning process that allows agents to choose an action among several possible actions. Because our protocol is defined as a set of conversation policies, this protocol has the characteristic to be more flexible than the traditional protocols such as those used in FIPA-ACL. This flexibility results from the fact that these policies can be combined to produce complete and more complex protocols. We formalized these conversation policies as a set of dialogue games, and we described the persuasion dynamics by the combination of five dialogue games. Another contribution of this paper is the tableau-based termination proof of the protocol. We also described the implementation of this protocol. Finally, we presented an example to illustrate the persuasion dynamics by the combination of different dialogue games.

As an extension of this work, we intend to specify other protocols according to Walton and Krabbe's classification [24] using the same framework. Another interesting direction for future work is verifying these protocols using model checking techniques. The method we are investigating is an automata theoretic approach based on a tableau method [9]. This method can be used to verify the temporal and dynamic aspects of our protocol.

Acknowledgements. We'd like to deeply thank the three anonymous reviewers for their valuable comments and suggestions. We'd also like to thank Rance Cleaveland and Girish Bhat for their interesting explanations on the tableau method.

\section{References}

1. Amgoud, L., Maudet, N., and Parsons, S. Modelling dialogues using argumentation. In Proc. of 4th Int. Conf. on Multi Agent Systems (2000) 31-38.

2. Amgoud, L., and Maudet, N. Strategical considerations for argumentative agents. In Proc. of 10th Int. Workshop on Non-Monotonic Reasoning (2002) 409-417.

3. Bentahar, J., Moulin, B., and Chaib-draa, B. Commitment and argument network: a new formalism for agent communication. In [12] (2003) 146-165.

4. Bentahar, J., Moulin, B., Meyer, J-J. Ch., and Chaib-draa, B. A logical model for commitment and argument network for agent communication (extended abstract). In $3^{\text {rd }}$ Int. J. Conf. on Autonomous Agents and Multi-Agent Systems AAMAS (2004) 792-799. 
5. Bentahar, J., Moulin, B., and Chaib-draa, B. A persuasion dialogue game based on commitments and arguments. In Proc. of Int. Workshop on Argumentation in Multi Agent Systems ArgMas (2004) 148-164.

6. Brewka, G. Dynamic argument systems: A formal model of argumentation processes based on situation calculus. Journal of Logic and Computation, 11(2) (2001) 257-282.

7. Castelfranchi, C. Commitments: from individual intentions to groups and organizations. In Proc. of Int. Conf. on Multi Agent Systems (1995) 41-48.

8. Chaib-draa, B., and Dignum, F. Trends in agent communication languages. In Computational Intelligence, (18)2 (2002) 89-101.

9. Cleaveland, R. Tableau-based model checking in the propositional mu-calculus. In Acta Informatica, 27(8) (1990) 725-747.

10. Colombetti, M. A commitment-based approach to agent speech acts and conversations. In Proc. of Int. Autonomous Agent Workshop on Conversational Policies (2000) 21-29.

11. Dastani, M., Hulstijn, J., and der Torre, L.V. Negotiation protocols and dialogue games. In Proc. of Belgium/Dutch AI Conference (2000) 13-20.

12. Dignum, F. (Ed.). Advances in Agent Communication. Int. Workshop on Agent Communication Languages. LNAI 2922, Springer, (2003).

13. Dignum, F., and Greaves, M. (Eds.). Issues in agent communication. LNAI 1916, Springer (2000).

14. Elvang-Goransson, M., Fox, J., and Krause, P. Dialectic reasoning with inconsistent information. In Proc. of $9^{\text {th }}$ Conf. on Uncertainty in Artificial Intelligence (1993) 114-121.

15. Endriss, U., Maudet, N., Sadri, F., and Toni, F. Logic_based agent communication protocols. In [12] (2003) 91-107.

16. Fornara, N. and Colombetti, M. Protocol specification using a commitment based ACL. In [12] (2003) 108-127.

17. Greaves, M., Holmback, H., and Bradshaw, J. What is a conversation policy? In [13] (2000) 118-131.

18. Maudet, N., and Chaib-draa, B. Commitment-based and dialogue-game based protocols, new trends in agent communication languages. In Knowledge Engineering Review, 17(2), Cambridge University Press (2002) 157-179.

19. McBurney, P., and Parsons, S. Games that agents play: A formal framework for dialogues between autonomous agents. In Journal of Logic, Language, and Information, 11(3) (2002) $1-22$.

20. Parsons, S., Wooldridge, M., and Amgoud, L. On the outcomes of formal inter-agent dialogues. In Proc. of $2^{\text {nd }}$ Int. J. Conf. on Autonomous Agents and Multi-Agent Systems (2003) 616-623.

21. Prakken, H. Relating protocols for dynamic dispute with logics for defeasible argumentation. In Synthese (127) (2001) 187-219.

22. Singh, M.P. A social semantics for agent communication language. In [13] (2000) 31-45.

23. The Agent Oriented Software Group. Jack 4.1. 2004. www.agent-software.com/

24. Walton, D.N., and Krabbe, E.C.W. Commitment in dialogue: basic concepts of interpersonal reasoning. State University of New York Press, NY (1995).

25. Yolum, P. and Singh, M.P. Flexible protocol specification and execution: applying event calculus planning using commitments. In Proc. of 1st Int. J. Conf. on Autonomous Agents and Multi-Agent Systems (2002) 527-534. 